\title{
Coherent excitonic nonlinearity versus inhomogeneous broadening in single quantum
} wells

Langbein, Wolfgang Werner; Borri, Paola; Hvam, Jørn Märcher; Birkedal, Dan

Published in:

Summaries of papers presented at the International Quantum Electronics Conference

Link to article, DOI:

10.1109/IQEC.1998.680119

Publication date:

1998

Document Version

Publisher's PDF, also known as Version of record

Link back to DTU Orbit

Citation (APA):

Langbein, W. W., Borri, P., Hvam, J. M., \& Birkedal, D. (1998). Coherent excitonic nonlinearity versus inhomogeneous broadening in single quantum wells. In Summaries of papers presented at the International Quantum Electronics Conference (pp. 55-56). IEEE. https://doi.org/10.1109/IQEC.1998.680119

\section{General rights}

Copyright and moral rights for the publications made accessible in the public portal are retained by the authors and/or other copyright owners and it is a condition of accessing publications that users recognise and abide by the legal requirements associated with these rights.

- Users may download and print one copy of any publication from the public portal for the purpose of private study or research.

- You may not further distribute the material or use it for any profit-making activity or commercial gain

- You may freely distribute the URL identifying the publication in the public portal 


\section{QTuG3}

\section{T-matrix approach for the optical spectra of semiconductor heterostructures}

M.F. Pereira Jr., T. Schmielau, R. Schepe, K. Henneberger, Fachbereich Physik, Universität Rostock Universitätsplatz 3, D18051 Rostock, Germany

Coulomb correlations are crucial for the realistic computation of semiconductor optical spectra. ${ }^{1}$ In low-dimensional systems, band structure and quantum confinement effects play also a major role and have to be integrated in the theory. In our approach, the excited semiconductor is described by nonequilibrium Green's functions for the interacting quasi-particles: carriers, photons, and plasmons, whose time evolution is governed by Dyson Equations. Coupled band structure and quantum confinement effects are included in the carrier propagators, and in the transition matrix elements, which give rise to optical selection rules, and e.g., TE/TM mode discrimination. They are determined after diagonalization of the Luttinger Hamiltonian. The emission and absorption spectra are computed from the transverse polarization function, $P$, i.e., the self-energy that appears in the photon Dyson equation. Both $P$ and the carrier selfenergy $\Sigma$, can be written as a sum of an RPA term and a Coulomb-correlation contribution, expressed by means of a T-matrix, which satisfies the Bethe-Salpeter equation. Figure 1 shows that for temperatures as low as $77 \mathrm{~K}$, the inclusion of beyond-RPA corrections in $\Sigma$ does not affect the computed spectra. All other curves are thus computed within this approximation. Figure 2 shows absorption spectra of a $50-\AA$ GaAs/AlGaAs QW, as well as corresponding luminescence spectra. Notably, to

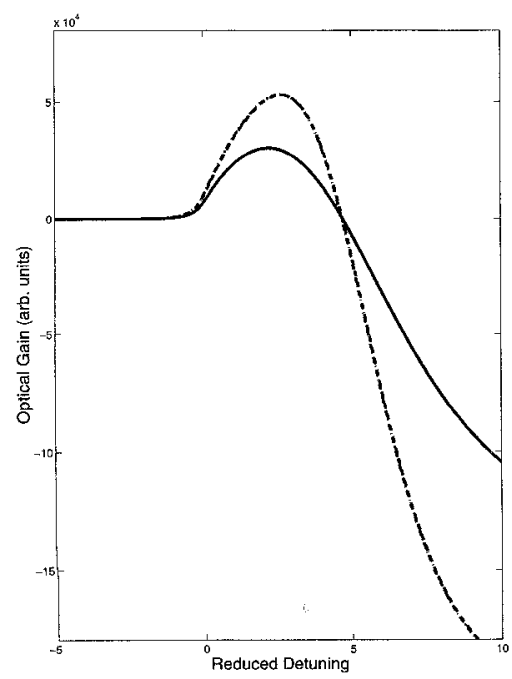

QTuG3 Fig. 1. TE (dashed) and TM (solid) absorption (a), with corresponding photoluminescence (PL) spectra, (b), for a $50 \AA$ GaAs $\mathrm{Al}_{0.23} \mathrm{Ga}_{0.77} \mathrm{As} \mathrm{QW}$ as a function of detuning with respect to the free-carrier bandgap at $77 \mathrm{~K}$. From top to bottom the carrier densities for absorption and PL are $N=0,0.1,0.5,0.75,1.0$ and $1.0,0.5$, $0.1 \times 10^{18}$ carriers $/ \mathrm{cm}^{3}$. The TM PL spectra have been multiplied by a factor $\approx 7.7$.
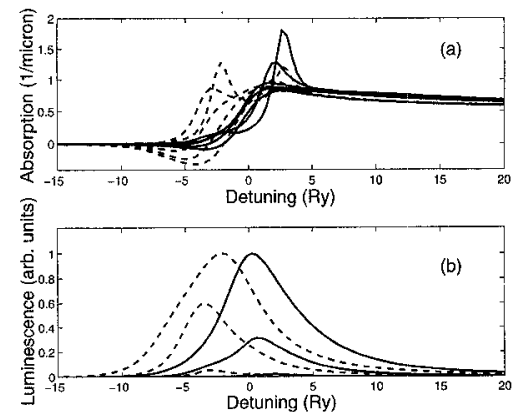

QTuG3 Fig. 2. TE-optical gain spectra for a $15 \AA / 75 \AA$ GaAs $-\mathrm{Al}_{0.23} \mathrm{Ga}_{0.77} \mathrm{As}$ superlattice, within the anisotropic medium approach of Ref. 2 , at $77 \mathrm{~K}$ and a carrier density of $\mathrm{N}=1.0 \times 10^{18}$ carriers $/ \mathrm{cm}^{3}$ as a function of reduced detuning. RPA bubble: solid; T-matrix corrections on the photon spectral density: dashed; T-matrix corrections on the photon and carrier spectral densities: dot-dashed. Only the E1-HH1 transition is shown.

the best of our knowledge for the first time, we predict a structure corresponding to a heavyhole in the TM spectra. It arises due to a combination of T-matrix (Coulomb) and bandcoupling effects, i.e., it can not be described by simple free-carrier or many-body approaches without valence band coupling.

Our theory consistently describes the evolution of the nonlinear absorption from the excitonic low-density regime to the gain region with correct line shapes. The spurious absorption below the gain region, found in simplified calculations, does not appear in our results, because we use a polarization function that satisfies the Kubo-Martin-Schwinger condition (KMS). Contributions from high-k values are then eliminated. We have thus an alternative and simpler approach to that introduced in Ref. 3, in which the problem is solved by means of nondiagonal dephasing terms, introduced within the context of coherent excitation described by semiconductor Bloch equations. In summary, we have presented a Green's function theory that allows the study of Coulomb correlations in the optical spectra of heterostructures. We have demonstrated that the major contribution that gives rise to excitonic spectra is due to the T-matrix in the polarization function, and obtained correct gain line shapes in a simple way by avoiding the KMS condition violation. We expect that $\mathrm{T}$ matrix contributions for the self-energy yield important deviations at very low temperatures and will investigate the effect, which requires a detailed numerical study of the fast-varying distribution functions, in forthcoming publications.

1. For a recent review see Microscopic Theory of Semiconductors: Quantum Kinetics, Confinement and Lasers, S.W. Koch, ed. (World Scientific, Singapore, 1995).

2. M.F. Pereira Jr., Phys. Rev. B 52, 1978 (1995).

3. S. Hugues et al., Solid State Commun. 100, 555 (1996).
QTuG4

Coherent excitonic nonlinearity versus inhomogeneous broadening in single quantum wells

W. Langbein, P. Borri, J.M. Hvam, D. Birkeda, ${ }^{*}$ Mikroelektronik Centret, The Technical University of Denmark, Building 345 east, DK-2800 Lyngby, Denmark;

E-mail: langbein@mic.dtu.dk

The coherent response of excitons in semiconductor nanostructures, as measured in fourwave mixing (FWM) experiments, ${ }^{1}$ depends strongly on the inhomogeneous broadening of the exciton transition. We investigate GaAs/ $\mathrm{Al}_{0.3} \mathrm{Ga}_{0.7}$ As single quantum wells (SQW) of 4 $\mathrm{nm}$ to $25 \mathrm{~nm}$ well width. Two main mechanisms are found to be important.

First, the excitation-induced dephasing FWM signal (EID), ${ }^{2}$ which leads to a strong dependence of the signal on the angle between the linear input polarizations. The presence of the EID in the mainly homogeneously broadened sample $(25 \mathrm{~nm})$ is shown by the beating between EID and two-photon coherence (TPC) at the exciton for negative delay [Fig. 1(a)] and co-linear polarized excitation $(\uparrow, \uparrow)$. It vanishes for cross-polarized excitation $(\uparrow, \rightarrow)$, thus excluding the importance of local-field effects.

The EID changes its character in an inho-
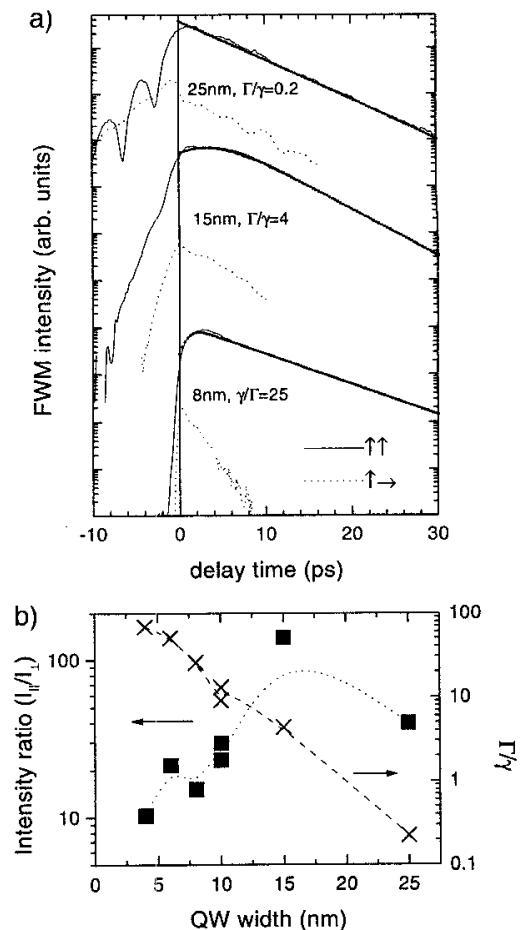

QTuG4 Fig. 1. a) Time-integrated FWM intensity at the exciton transition energy for colinear (solid line) and cross-linear (dotted line) polarized excitation in GaAs SQW of different widths as indicated. Dots: fitted behavior for PSF in presence of inhomogeneous broadening. The ratio between inhomogeneous $\Gamma$ and homogeneous $\gamma$ broadenings is given. b) Signal intensity ratio for co-linear and cross-linear excitation at zero delay time, and $\Gamma / \gamma$ as a function of well width. 

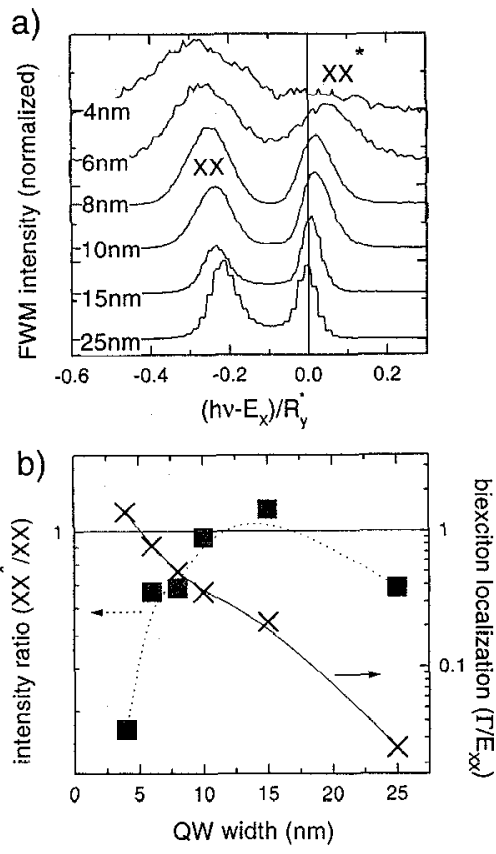

QTuG4 Fig. 2. a) Normalized, spectrally resolved FWM intensity at a delay of $h / E_{\mathrm{Xx}}\left(E_{\mathrm{Xx}}\right.$ : biexciton binding energy) for cross-linear polarization. The energy axis is offset and scaled with the respective exciton transition energy $E_{\mathrm{X}}$ and exciton binding energy $R_{y}^{*}$, b) Ratio between the FWM intensity at the $\mathrm{XX}$ and $\mathrm{XX}^{*}$ transitions, as deduced from the spectra in a).

mogeneous system, because the macroscopic density grating vanishes due to destructive interference, and no photon echo (PE) is created. ${ }^{3}$ However, the observed signal intensity ratio between $(\uparrow, \rightarrow)$ and $(\uparrow, \uparrow)$ even increases for a small inhomogeneous broadening [Fig. 1(b)]. A persistence of the EID is expected for a dephasing related only to microscopic densities ${ }^{4}$ showing a quadratic delay-time dependence relative to the phase-space filling FWM signal (PSF). However, the delay-time dependence of the time-integrated $(\uparrow, \uparrow)$ FWM signal at the exciton transition matches the expected response of the PSF in the inhomogeneous case, ${ }^{5}$ as demonstrated by the fitted curves [Fig. 1(a)]. We thus have to conclude that the response of the system is in between the two mentioned characters of the EID. Indeed, assuming a density-dependent dephasing rate with a coefficient $\gamma^{\prime}(\Delta E)$ scaling with the energy difference $\Delta E^{-1}$ between two subsystems in the inhomogeneous distribution (as expected from perturbation theory), an EID dependence on the delay time like the PSF can be obtained. For large inhomogeneous broadening, the EID contribution decreases [Fig. 1(b)]. This is related to the smaller exciton density of states $\mathrm{D}(\mathrm{E})$ and a smaller wave-function overlap.

We now turn to the second mechanism, that is effecting the $(\uparrow, \rightarrow)$ FWM response. In this case the signal for positive delay is dominated by PSF. It has been pointed out ${ }^{6}$ that the signal is given by the transitions from a oneexciton state $\mathrm{X}$ to two-exciton states $2 \mathrm{X}$. The $2 \mathrm{X}$ spectrum can be modeled by a bound (XX) and an unbound $\left(\mathrm{XX}^{*}\right)$ biexciton state. This has two implications. First, the dephasing of the third-order polarization is given by the sum of the dephasing of $X$ and $2 X$. Second, in the inhomogeneous case, the rephasing of the microscopic third-order polarization to the macroscopic FWM signal is blurred by the nonperfect correlation of $\mathrm{X}$ and XX energies. ${ }^{7}$ This leads to the faster and nonexponential signal decay in delay time [Fig. 1(a)]. The X-2X spectrum is visible in the $(\uparrow, \uparrow)$ FWM spectrum [Fig. 2(a)]. For an inhomogeneous broadening $\Gamma$ small compared to the biexciton binding energy $E_{\mathrm{XX}}$, both transitions have comparable strength, and are shifting opposite by the same amount. For $\Gamma \approx E_{\mathrm{XX}}$, the $\mathrm{XX}^{*}$ is suppressed [Fig. 2(b)] due to the quantization of the biexcitonic continuum in the localization potential. ${ }^{8}$

${ }^{*}$ Lucent Technologies, Bell Laboratories, 101 Crawfords Corner Road, Holmdel, New Jersey 07733

1. J. Shah, in Ultrafast Spectroscopy of Semiconductors and Semiconductor Nanostructures (Springer, Berlin, 1996), Chap. 2.

2. Y.Z. Hu et al., Phys. Rev. B 49, 14382 (1994).

3. H. Wang et al., Phys. Rev. B 49, R1551 (1994).

4. P. Borri et al., Phys. Status Solidi A, in press (1997)

5. J. Erland et al., Phys. Rev. B 50, 15047 (1994).

6. K. Bott et al., Phys. Rev. B 48, 17418 (1993).

7. W. Langbein et al., Phys. Rev. B 55, R7383 (1997).

8. W. Langbein and J.M. Hvam, Phys. Status Solidi $B$, in press.

\section{QTuG5}

Dephasing of Bloch oscillations due to carrier-carrier scattering: coherent versus incoherent scatterers

F. Wolter, R. Martini, S. Tolk, H.T. Grahn, * R. Hey, ${ }^{*}$ P. Haring Bolivar, H. Kurz, Institut für Halbleitertechnik II, RWTH Aachen, Sommerfeldstr. 24, D-52056 Aachen, Germany

The practical application of Bloch oscillations in semiconductor superlattices as $\mathrm{THz}$ emitters is hampered by the low emission power and the rapid dephasing of the coherent signal. ${ }^{1}$ At higher excitation densities the quadratic scaling of the $\mathrm{THz}$ emission power saturates due to the stronger dephasing of the signal. We adopt an approach previously employed for the investigation of the dephasing of interband dynamics in four wave mixing ${ }^{2}$ to study the dephasing of the intraband dynamics responsible for the THz emission. We demonstrate that for higher carrier densities the dominant dephasing process at low temperatures is carrier-carrier scattering. In addition, the presence of incoherent relaxed background carriers generated by a prepulse lead to a stronger dephasing than in the case where the same amount of coherent scatterers are created. The experiments are performed on a superlattice consisting of 9.7-nm GaAs wells separated by $1.7-\mathrm{nm} \mathrm{Al} \mathrm{l}_{0.3} \mathrm{Ga}_{0.7}$ As barriers with a calculated width of the first electron miniband of $18 \mathrm{meV}$. In order to apply an electric field in the growth

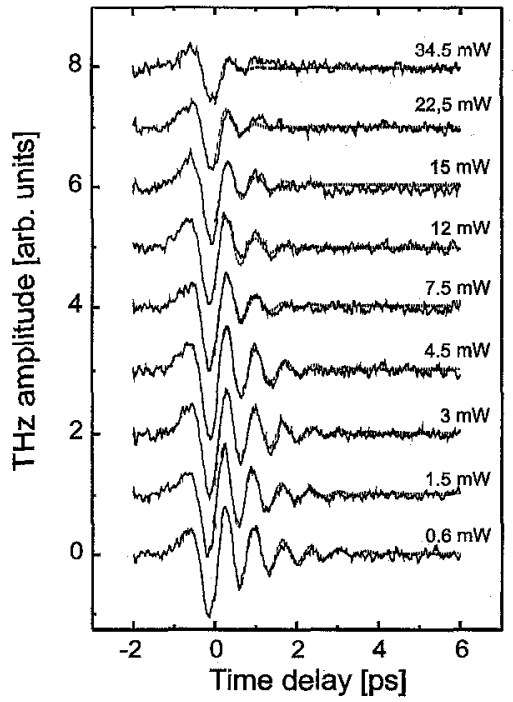

QTuG5 Fig. 1. THz transients for different densities of the incoherent carrier background. For increasing densities the damping increases. The fits reveal a single-exponential decay of the amplitude.

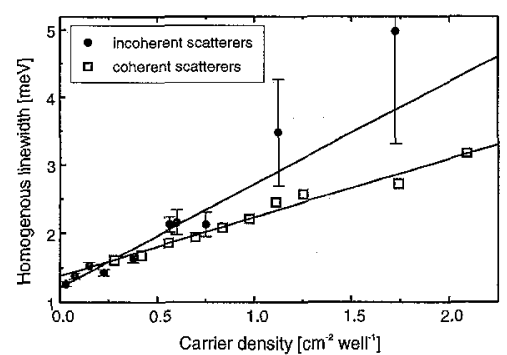

QTuG5 Fig. 2. Homogenous linewidth vs. excitation density. From linear regression the interaction parameter can be derived. The excitation of incoherent scatteres (solid circles) leads to a stronger dephasing than a comparable density of coherent scatterers (open squares).

direction the structure is embedded in the intrinsic region of a pin-diode. The sample is held at $20 \mathrm{~K}$ in a closed-cycle cryostat. The coherent dynamics is probed by $\mathrm{THz}$ emission spectroscopy. A prepulse 20 ps before the exciting pulse creates a cold background plasma. The long delay makes sure that the coherent motion of the carriers is completely dephased. Figure 1 displays $\mathrm{THz}$ transients for different intensities of the pre-pulse. Clearly, a higher carrier density leads to a stronger damping of the Bloch oscillation. A single exponentially damped cosine function fitted to the transients allows to determine the dephasing time $\tau$, which corresponds to a homogenous linewidth $\Gamma=h / \pi \tau$. The fit procedure is restricted to longer time delays to avoid instantanous contributions. The linewidth shown in Fig. 2 (solid circles) reveals a linear dependency on the excitation density according to $\Gamma(n)=$ $\Gamma(0)+\gamma n_{\text {exc }}, \Gamma(0)=1.25 \mathrm{meV}$ accounts for the density-independent linewidth, i.e., due to interface roughness as well as inhomogenous broadening. The slope $\gamma_{\text {incoh }}=1.5^{*} 10^{-11} \mathrm{~cm}^{2}$ well is a measure for the influence of carriercarrier scattering on the dephasing. The domi- 